\title{
Simvastatin and ROCK inhibition in Th0 and Th17 systems
}

\author{
Josephine Isgro ${ }^{2 *}$, Li Song ${ }^{1}$, Sanjay Gupta ${ }^{1}$, Alessandra B Pernis ${ }^{1}$ \\ From 2011 Pediatric Rheumatology Symposium sponsored by the American College of Rheumatology \\ Miami, FL, USA. 2-5 June 2011
}

\section{Purpose}

The deregulation of the Def6-ROCK2-IRF4 axis in murine models results in both lupus-like and rheumatoid arthritis-like disease characterized by increased IL-17 and IL-21 production that is ameliorated by ROCK inhibition. A known beneficial pleiotropic effect of statins is inhibition of ROCK activation via their effect on RhoA activation. Statins have also been reported to decrease IL-17 and IL-21 production in T cells. Whether statins exert their inhibitory effects by interfering with the ROCK2-IRF-4 interaction in CD4+ T cells is unknown. The aim of the present study is to investigate whether statins can inhibit the ROCK pathway in CD4+ T cells and inhibit IL-17 and IL-21 production.

\section{Methods}

Purified CD4+ T cells from the spleens and lymph nodes of wild type and Def6-deficient DO11.10 transgenic mice were stimulated with $\alpha \mathrm{CD} 3$ and $\alpha \mathrm{CD} 28$ in the presence/ absence of simvastatin $(1-10 \mu \mathrm{M})$ with and without the known ROCK inhibitor, Y-27632 (10-30 $\mu$ M). Supernatants were collected and IL-17 and IL-21 production analyzed by ELISA.

\section{Results}

As previously reported, Def6-deficient CD4+ T cells secreted significantly higher levels of IL-17 and IL-21 when stimulated as compared to wild type controls, which was ameliorated by addition of the ROCK inhibitor, Y27632. Simvastatin significantly decreased the concentrations of IL-17 and IL-21 at all tested concentrations in a dose dependent manner $(\mathrm{p}<.05)$. At lower concentrations of simvastatin $(<2.5 \mu \mathrm{M})$, the addition of Y-27632 further decreased cytokine production.

\section{Conclusion}

These data suggest that simvastatin can interfere with the ROCK pathway in CD4+ T cells and inhibit IL-17 and IL21 production in a murine model of autoimmunity. As statins and ROCK inhibitors have distinct targets, our data furthermore suggest that combination therapy with a statin and a ROCK inhibitor may be more effective than monotherapy. Furthermore, we speculate that the decrease in cytokines is linked to a decrease in the phosphorylation status of IRF4 and its ability to target the promoters of these cytokines.

\section{Disclosure}

Josephine Isgro: None; Li Song: None; Sanjay Gupta: None; Alessandra B. Pernis: None.

\section{Author details}

'Hospital for Special Surgery, New York, NY, USA. ²Morgan Stanley Children's Hospital of New York Presbyterian, Columbia University Medical Center, New York, NY, USA.

Published: 13 July 2012

doi:10.1186/1546-0096-10-S1-A112

Cite this article as: Isgro et al:: Simvastatin and ROCK inhibition in Tho and Th17 systems. Pediatric Rheumatology 2012 10(Suppl 1):A112. 